\title{
DIFFERENTIAL APPROXIMATION OF NP-HARD PROBLEMS WITH EQUAL SIZE FEASIBLE SOLUTIONS
}

\author{
JÉRÔME MONNOT
}

LAMSADE, Université Paris-Dauphine, Place du Maréchal de Lattre de Tassigny, 75775 Paris Cedex 16, France.

monnot@lamsade. dauphine.fr

\begin{abstract}
In this paper, we focus on some specific optimization problems from graph theory, those for which all feasible solutions have an equal size that depends on the instance size.

Once having provided a formal definition of this class of problems, we try to extract some of its basic properties; most of these are deduced from the equivalence, under differential approximation, between two versions of a problem $\pi$ which only differ on a linear transformation of their objective functions. This is notably the case of maximization and minimization versions of $\pi$, as well as general minimization and minimization with triangular inequality versions of $\pi$. Then, we prove that some well known problems do belong to this class, such as special cases of both spanning tree and vehicles routing problems. In particular, we study the strict rural postman problem (called SRPP) and show that both the maximization and the minimization versions can be approximately solved, in polynomial time, within a differential ratio bounded above by $1 / 2$.

From these results, we derive new bounds for standard ratio when restricting edge weights to the interval $[a, t a]$ (the $S R P P[t]$ problem): we respectively provide a $2 /(t+1)$ - and a $(t+1) / 2 t$-standard approximation for the minimization and the maximization versions.

Keywords: Approximate algorithms; Differential ratio; Performance ratio; Analysis of Algorithms;
\end{abstract}

\section{Introduction}

Routing design problems are of major importance in combinatorial optimization, and related works have been numerous during the last twenty years (see Christofides [7], Fisher et al. [18], Kosaraju et al. [35], Engebretsen and Karpinski [17]). The purpose of this paper is to study a class of graph-theoretic problems, called FGNPO ${ }^{1}$, including Depth-constrained Spanning Tree and Traveling Salesman problems. The feasible solutions of each problem of $F G N P O$ are subsets of edges having an equal size (depending on the instance), and the cost of a solution is the sum of the weights on its edges. For example, the minimum spanning tree problem is typically an FGNPO problem: each tree has exactly $n-1$ edges and the cost of a solution is the sum of the weights on the tree's edges. On the other hand, the minimum Steiner tree problem is not in FGNPO since the number of edges in a Steiner tree is not uniquely determined by the input size. Other close problem families (sharing the fixed solution size property) have been widely studied over the past ten years; for instance, the problems of finding minimum-cost subsets of a combinatorial structure and a given size are the most common (see Aggarwal et al. [1], Ravi et al. [46]). In particular, concerning the problem Min $k-M S T$, the performance ratio has been recently improved to the best possible for general graphs and Euclidean graphs, (see Garg [21] and Mitchell [38]). Also problems of finding subsets maximizing minimum structures such as Remote $k-M S T$ and Remote $k-T S P$ have been studied by Halldorsson et al. [26].

\footnotetext{
${ }^{1}$ Fixed-size-solution Graph NPO.
} 
We observe an important fact on these problems: the fixed-size property of solutions induces an homogeneous complexity between maximization and minimization versions since both versions of each problem are equivalent from an optimization point of view (i.e., they are Karp-reducible [32]).

We focus on the design of approximation algorithms with guaranteed performance ratios. Usually, one compares the worst-case ratio (here called standard ratio) of the cost of the solution generated by the algorithm to the optimal cost. However, we mainly refer in this article to another ratio called differential ratio which measures the worst ratio of, on the one hand, the difference between the cost of the solution generated by the algorithm and the worst cost, and on the other hand, the difference between the optimal cost and the worst cost. This measure, studied by Aiello et al. [2], Ausiello et al. [6], Fisher et al. [18], Vavasis [48] (in the context of non-linear programming), Zemel [49] and more recently by Demange and Paschos [15] and Hassin and Khuller [27], leads to new algorithms taking into account the extreme solutions of the instance, and provides the opportunity to better understand these problems. In this paper, we show that there are tight links between both measures for the studied problems. Most of the positive approximation results are obtained by getting, for each problem, several feasible solutions $x_{1}, \ldots, x_{q}$ and by choosing the best one among them; each of these individual solutions has a differential approximation ratio tending towards zero when the instance size increases. In other words, for each solution $x_{i}$, there exists a subset of instances $S_{i}$ (called Family of critical instances in Monnot [39]) illustrating this worst-case behavior. Our approximate algorithms have the property that, for each instance in $S_{i}$, there is another solution $x_{j}$ which has a good approximation ratio. These notions of families of critical and strongly critical instances are well known in the polynomial approximation community, but they have never been finalized until now. However in Monnot [39], they have been largely studied and commented.

We now give some standard definitions in the field of optimization and approximation theory. For a more detailed statement of this theory, we refer the reader to Ausiello et al. [5], [4], Hochbaum [29].

Definition 1.1. An NP-optimization problem (NPO-problem) $\pi$ is a five-tuple $(D$, sol, $m, T r i v, g o a l)$ such that:

(i) $D$ is the set of instances and is recognizable in polynomial-time.

(ii) Given an instance $I \in D, \operatorname{sol}[I]$ is the set of feasible solutions of $I$; moreover, there exists a polynomial $P$ such that, for any $x \in \operatorname{sol}[I],|x| \leq P(|I|)$; furthermore, it is decidable in polynomial time whether $x \in \operatorname{sol}[I]$ for any $I$ and for any $x$ such that $|x| \leq P(|I|)$. Finally, there is a feasible solution Triv $(I)^{2}$ computable in polynomial-time for any $I$.

(iii) Given an instance $I$ and a solution $x$ of $I, m[I, x]$ denotes the positive integer value of $x$. The function $m$ is computable in polynomial time and is also called the objective function.

(iv) goal $\in\{\operatorname{Max}, \operatorname{Min}\}$.

We call $\overline{g o a l}^{3}$ the complementary notion of goal and $\bar{\pi}$ the NPO-problem $(D$, sol, m, Triv, $\overline{\text { goal }})$. The goal of an NPO-problem with respect to an instance $I$ is to find an optimum solution $x^{*}$ such that $O P T(I)=m\left[I, x^{*}\right]=\operatorname{goal}\{m[I, x]: x \in \operatorname{sol}[I]\}$. Another important solution of $\pi$ is a worst solution $x_{*}$ defined by: $\operatorname{WOR}(I)=m\left[I, x_{*}\right]=\overline{\operatorname{goal}}\{m[I, x]: x \in \operatorname{sol}[I]\}$. A worst solution for $\pi$ is an optimal solution for $\bar{\pi}$ and vice versa. In Ausiello et al. [6], the term trivial solution referred to as worst solution and all the exposed examples have the property that a worst solution can be trivially computed in polynomial-time. For example, this is the case of the maximum cut problem where, given a graph, the worst solution is the empty edge-set, and the bin-packing problem where we can trivially put the items using a distinct bin per item. It is not the case however in this paper, where the computation of a worst solution is far from being trivial.

\footnotetext{
${ }^{2}$ The common definition of class NPO does not require the existence of a trivial solution.

${ }^{3}$ If $g o a l=M a x$, then $\overline{\text { goal }}=$ Min and $\overline{\overline{\text { goal }}}=\operatorname{Max}$.
} 
1.1. Approximate algorithms and reductions. In order to study algorithm performances, there are two known measures: standard ratio, see Garey and Johnson [20], Ausiello et al. [5], Crescenzi and Kann [9] and differential ratio, see Demange and Paschos [15], Ausiello et al. [6], Cornuejols et al. [8], Zemel [49].

Definition 1.2. Let $\pi$ be an $N P O$ problem and $x$ be a feasible solution of an instance $I$. We define the performance ratios of $x$ with respect to the instance $I$ as

- (standard ratio) $\rho[\pi](I, x)=\operatorname{Min}\left\{\frac{m[I, x]}{O P T(I)}, \frac{O P T(I)}{m[I, x]}\right\}$

- (differential ratio) $\delta[\pi](I, x)=\frac{W O R(I)-m[I, x]}{W O R(I)-O P T(I)}$

The performance ratio is a number less than or equal to 1 , and is equal to 1 if and only if $m[I, x]=$ $O P T(I)$. Remark that we have inverted the usual standard performance ratio in the case of minimization problems so that the ratio value is always between 0 and 1 . Let $\pi$ be an $N P O$ problem. For any instance $I$ of $\pi$, a polynomial time algorithm $A$ returns a feasible solution $x^{A}$. The performance of $A$ with respect to $R \in\{\delta, \rho\}$ on the instance $I$ is the quantity $R_{A}[\pi](I)=R[\pi]\left(I, x^{A}\right)$. We say that $A$ is an $r$-approximation algorithm with respect to $R$ if for any instance $I$, we have $R_{A}[\pi](I) \geq r$.

Definition 1.3. For any performance ratio $R \in\{\delta, \rho\}$,

- an $N P O$ problem belongs to the class $A P X(R)$ if there exists an $r$-approximation algorithm with respect to $R$ for some constant $r \in] 0 ; 1]$.

- an $N P O$ problem belongs to the class $P T A S(R)$ if there exists an $r$-approximation algorithm $A_{r}$ for any constant $r \in] 0 ; 1\left[\right.$. The family $\left\{A_{r}\right\}_{0<r<1}$ is said to be an approximation scheme.

- an NPO problem belongs to the class FPTAS(R) where $R \in\{\delta, \rho\}$ if there exists a $r$-approximation algorithm with respect to $R$, for any constant $r \in] 0 ; 1[$ and the time-complexity of this algorithm is $O\left[P\left(|I| ; \frac{1}{1-r}\right)\right]$ where $\mathrm{P}$ is a polynomial of two variables.

Clearly, the following inclusions hold for any performance ratio $R \in\{\delta, \rho\}: F P T A S(R) \subseteq P T A S(R) \subseteq$ $A P X(R)$. As it is usually done, we will denote by $A P X, P T A S$ and FPTAS, respectively, the classes $A P X(\rho), P T A S(\rho)$ and $F P T A S(\rho)$. As shown in Demange et al. [12], many problems such as the Vertex Covering or the Dominating Set problems can have different behavior patterns depending on whether the differential or standard ratio is chosen. On the other hand, for several problems we may establish some connections between the differential and the standard ratios, like Bin Packing (see Demange et al. [13]) or Steiner Tree problem with distance one and two (see Demange et al. [14]) and see Zemel [49] for motivations and complementarity links between the two measures. Besides for the problems that we consider here, we show that there are tight links between the two measures when the ratio $d_{\max } / d_{\min }$ is upper bounded (where $d_{\max }$ and $d_{\min }$ denote respectively the maximum and the minimum cost of the edges).

Consider the following approximation preserving reductions between pairs $(\pi, R)$ :

Definition 1.4. For $\pi_{i} \in N P O$ and $R_{i} \in\{\delta, \rho\}, i=1,2$,

- an A-reduction from the pair $\left(\pi_{1}, R_{1}\right)$ to the pair $\left(\pi_{2}, R_{2}\right)$, denoted by $\left(\pi_{1}, R_{1}\right) \leq{ }^{A}\left(\pi_{2}, R_{2}\right)$, is a triplet $(\propto, f, c)$ such that:

(i) $\propto: D_{\pi_{1}} \longmapsto D_{\pi_{2}}$, transforms an instance of $\pi_{1}$ into an instance of $\pi_{2}$ in polynomial time.

(ii) $f: \operatorname{sol}_{\pi_{2}}[\propto(I)] \longmapsto \operatorname{sol}_{\pi_{1}}[I]$, transforms solutions for $\pi_{2}$ into solutions for $\pi_{1}$ in polynomial time.

(iii) $c:[0 ; 1] \longmapsto[0 ; 1]$ (called expansion of the A-reduction) is a function verifying $c^{-1}(0) \subseteq\{0\}$, and $\forall \varepsilon \in[0 ; 1], \forall I \in D_{\pi_{1}}, \forall x \in \operatorname{sol}_{\pi_{2}}[\propto(I)]: R_{2}\left[\pi_{2}\right](\propto(I), x) \geq \varepsilon \Longrightarrow R_{1}\left[\pi_{1}\right](I, f(x)) \geq c(\varepsilon)$

- an $A * P$-reduction from the pair $\left(\pi_{1}, R_{1}\right)$ to the pair $\left(\pi_{2}, R_{2}\right)$, denoted by $\left(\pi_{1}, R_{1}\right) \leq^{A * P}\left(\pi_{2}, R_{2}\right)$, is an $A$-reduction from $\left(\pi_{1}, R_{1}\right)$ to $\left(\pi_{2}, R_{2}\right)$ such that the restriction of function $c$ to an interval $[a ; 1]$ is bijective and $c(1)=1$ (note that $c$ does not necessarily verify $c^{-1}(0) \subseteq\{0\}$ ). 
Definition 1.5. If $\left(\pi_{1}, R_{1}\right) \leq^{A * P}\left(\pi_{2}, R_{2}\right)$ and $\left(\pi_{2}, R_{2}\right) \leq^{A * P}\left(\pi_{1}, R_{1}\right)$ with $c(\varepsilon)=\varepsilon$, we say that $\left(\pi_{1}, R_{1}\right)$ is equivalent to $\left(\pi_{2}, R_{2}\right)$.

The $A$-reduction preserves constant ratio approximation while the $A * P$-reduction preserves approximation schemes. They are natural generalizations of reductions described by Orponen and Mannila [45], Ausiello et al. [5], Crescenzi and Panconesi [10].

The differential ratio measures how the value of an approximate solution is located in the interval between $W O R(I)$ and $O P T(I)$ whereas for a maximization problem, the standard ratio measures how the value of an approximate solution is placed in the interval between 0 and $O P T(I)$. Hence in this case, we have an $A * P$-reduction from the standard ratio to the differential ratio :

Lemma 1.6. If $\pi=(D$, sol, $m$, Triv, Max $) \in N P O$, then $(\pi, \rho) \leq^{A * P}(\pi, \delta)$ with $c(\varepsilon)=\varepsilon$.

Proof : Let $I$ be an instance of $\pi$ and $x$ be a feasible solution. If $m[I, x] \geq \varepsilon O P T(I)+(1-\varepsilon) W O R(I)$ then $m[I, x] \geq \varepsilon O P T(I)$ since $W O R(I) \geq 0$.

Remark that, in general, there is no evident transfer of a positive or negative result from one framework to the other for a minimization problem. For instance, we have proved in Demange et al. [11] that the best achievable standard ratio is $7 / 8$ for a version of weighted minimum coloring in bipartite graphs whereas we have built a differential approximation scheme; in this coloring version, the cost of a stable set is given by the maximum of the vertex weights in this stable set.

The rest of this paper is organized as follows. In section 2, we give a general definition of the FGNPO class and some elementary properties are presented. In section 3, we study the approximation of depthconstrained Spanning Tree problems. In section 4, we give approximation results of strict rural postman problems and some results are given for derived problems, namely the traveling salesman, Hamiltonian path with specified endpoints and clustered traveling salesman. Finally, in section 5, we conclude by some prospects in order to obtain negative differential results on the FGNPO class.

\section{The FGNPO problems}

We study a class of graph NPO-problems having the property that for any couple $(x, y)$ of feasible solutions, the size of $x$ and $y$ are the same (i.e., $|x|=|y|)$; the feasible solutions are subsets of edges of equal cardinality and the cost of a solution is the sum of the weights on its edges. Many well-known NP-hard problems are in this class as the Traveling Salesman problem or tree-problems with additional constraints (see Magnanti and Wolsey [36]) but also all the problems of partitioning into a given sub-graph (an approximation algorithm is given in Hassin and Rubinstein [28] when the sub-graph is 3-edge path). The latter problems called $H$-partition in Kirkpatrick and Hell [33], can be formulated as follows: a $H$-partition of a complete graph $K_{n}$ with edges valued by a function $d$ is a set of sub-graphs $\left(G_{1}, \ldots, G_{k}\right)$ where $G_{i}=\left(V_{i}, E_{i}\right)$ such that each $G_{i}$ is isomorphic to $H$ and the $\operatorname{set}(\mathrm{s}) V_{1}, \ldots, V_{k}$ partition $V$; the goal is to optimize $\sum d\left(E_{i}\right)$.

Formally, we can define these problems in the following way:

Definition 2.1. Let $\pi$ be an NPO problem; $\pi$ is called $F G N P O^{4}$ if it satisfies the following properties:

(i) An instance $I$ is a complete graph $K_{n}=(V, E)$, edge-valued by a function $d: E \rightarrow \mathbb{N}$.

(ii) A feasible solution is a subset $E^{\prime} \subseteq E$ of edges such that pred $\left(E^{\prime}\right)$ is true, where pred is a suitable polynomial-time decidable predicate. Moreover, there exists an integer function $g$ such that $\left|E^{\prime}\right|=g(|V|)$.

(iii) The objective function verifies $m\left[I, E^{\prime}\right]=\sum_{e \in E^{\prime}} d(e)$.

\footnotetext{
${ }^{4}$ Fixed-size-solution Graph NPO.
} 
If goal $=\operatorname{Max}$, the problem is called $\operatorname{Max} \pi$, else Min $\pi$. We use notation $\pi$ with no prefix when we consider without distinction the case goal $=$ Max or goal $=$ Min. Finally, $\pi[t]$ for some $t>1$ indicates the sub-problem verifying $d_{\max } \leq t d_{\min }$ where $d_{\max }=\max _{e \in E} d(e)$ and $d_{\min }=\min _{e \in E} d(e)$. For sake of simplicity, we identify $\pi$ with $\pi[\infty]$.

The FGNPO-problems verify the following properties:

For any $t>1, \pi[t] \in F G N P O$ is $N P$ - hard if and only if so is $\overline{\pi[t]}$ where we remember that $\bar{\pi}=(D, S, m$, Triv, Min $)$ when $\pi=(D, S, m$, Triv, Max $)$ and $\overline{\bar{\pi}}=\pi$; in other words, computing a worst solution of $\pi[t]$ is as hard as computing an optimal one. When $\pi[t]$ is $N P$ - hard for some constant $t>1$, Max $\pi[t]$ and Min $\pi[t]$ are trivially in $A P X$ since any solution is at least a $1 / t$-standard approximation; in this case, the standard ratio may not be very meaningful since even a worst solution yields a constant standard approximation. On the other hand, when $t$ tends towards infinity (in other words, we are interested in the general problem), the standard approximation results are very different for the two versions (maximization and minimization); $\operatorname{Max} \pi$ seems to be usually in $A P X$ (we do not know a problem $\pi \in F G N P O$ such that $\operatorname{Max} \pi \notin A P X)$ whereas generally, the problems Min $\pi$ are not in $A P X$. This last assertion is a natural generalization which comes from the well-known result established by Sahni and Gonzalez [47] for TSP: if Min $\pi \in A P X$, then the following decision problem is polynomial. Given a simple graph $G=(V, E)$, does $E^{\prime} \subseteq E$ exist such that $\left|E^{\prime}\right|=g(|V|)$ and $\operatorname{pred}\left(E^{\prime}\right)$ ? (we call it the decision problem associated to $\pi$ )

Proposition 2.2. Let $\pi$ be an FGNPO problem. If the decision problem associated to $\pi$ is NP-Complete then $M$ in $\pi$ is not in $A P X$ unless $P=N P$.

Proof : The proof of this claim is similar to the one in Sahni and Gonzalez [47]. Suppose that the algorithm $A$ yields a $\varepsilon$-standard approximation for Min $\pi$. We could decide if a graph $G$ verifies the property $P$ by the following way: From $G=(V, E)$, we build the instance $I=\left(K_{n}, d\right)$ of $\pi$ by taking $d(e)=1$ if $e \in E$ else $d(e)=\left\lceil\frac{g(|V|)}{\varepsilon}\right\rceil$ and we evaluate the quantity $A(I)$; we affirm that $G$ verifies $P$ if and only if $A(I) \leq \frac{g(|V|)}{\varepsilon}$. Indeed, if the graph $G$ verifies $P$ then by construction $O P T(I)=g(|V|)$ and $A(I) \leq \frac{O P T(I)}{\varepsilon}=\frac{g(|V|)}{\varepsilon}$. Conversely, if the graph $G$ does not verify $P$, then $A(I) \geq O P T(I)>\frac{g(|V|)}{\varepsilon}$ since we can easily suppose that $g(n) \geq 2$.

We deduce that $\operatorname{Min} T S P, \operatorname{Min} H P P_{s, p}{ }^{5}$, Min $H-$ Partitioning are not in $A P X$ unless $P=N P$, because the associated decision problems are $N P$-Complete. The reverse is not true since the decision

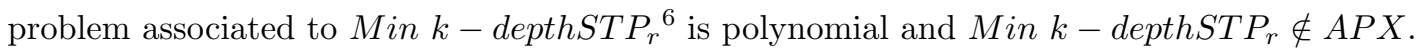

This asymmetry in the approximability of both versions can be considered as somewhat strange given the structural symmetry existing between them. Since differential approximation is stable under affine transformation of the objective function, $\operatorname{Max} \pi$ and $\operatorname{Min} \pi$ are differential-equivalent ${ }^{7}$. Besides, another difference with the standard ratio is that $\pi$ is not more difficult than the same problem with triangular inequality (called metric $\pi$ ). Finally, for the restriction where the weight function is bivalued (called $\pi(a, b))$, we can always boil down to the case $a=1$ and $b=2$.

Proposition 2.3. For a problem $\pi \in F G N P O$, the following assertions hold:

(i) For any $t \in] 1 ; \infty]$, Min $\pi[t]$ is differential-equivalent to Max $\pi[t]$.

(ii) $\pi$ is differential-equivalent to metric $\pi$.

(iii) $\pi(a, b)^{8}$ is differential-equivalent to $\pi(1,2)$.

\footnotetext{
${ }^{5}$ Hamiltonian Path with specified endpoints $s$ and $p$.

${ }^{6} k$-depthSTP $P_{r}$ consists in finding a Tree $T$ where the length of any path in $T$ from $r$ is at most $k$.

${ }^{7}$ See definition 1.5 .

${ }^{8}$ Restriction where all edge weights are $a$ or $b$.
} 


\section{Proof :}

- For (i): Let $I=(G, d)$ be an instance of $\pi[t]$, we transform $I$ into instance $\propto(I)=\left(G, d^{\prime}\right)$ of $\bar{\pi}$ defined by: $\forall e \in E, d^{\prime}(e)=d_{\max }+d_{\min }-d(e)$. We observe that $\propto(I)$ is also an instance of $\pi[t]$. We obtain for any feasible solution $E^{\prime}: m\left[\propto(I), E^{\prime}\right]=g(|V|)\left(d_{\max }+d_{\min }\right)-m\left[I, E^{\prime}\right]$. Since $g(|V|)\left(d_{\max }+d_{\min }\right)$ is constant, the differential ratio is the same for $\pi[t]$ and $\overline{\pi[t]}$ (which also holds for the proofs of $(i i)$ and (iii).

- For (ii), the proof is similar, except that function $d^{\prime}$ is now defined by $d^{\prime}(e)=d_{\text {max }}+d(e)$. We remark that $d^{\prime}$ satisfies the triangle inequality and we have for any solution $E^{\prime}: m\left[\propto(I), E^{\prime}\right]=g(|V|) d_{\max }-$ $m\left[I, E^{\prime}\right]$

- Finally, for (iii), we consider $\propto(I)$ of $\pi(1,2)$ defined by: $\propto(I)=\left(G, d^{\prime}\right)$ with $d^{\prime}(e)=1$ iff $d(e)=a$. We obtain for any solution $E^{\prime}: m\left[\propto(I), E^{\prime}\right]=\frac{m\left[I, E^{\prime}\right]-(2 a-b) g(|V|)}{b-a}$

All the conditions defining the FGNPO class are important for establishing the previous properties, not only the fixed-size conditions. Some problems with fixed-size solutions are not in FGNPO and do not verify the differential-equivalence properties of proposition 2.3. For example, Min $k-M S T$, (see Ravi et al. [46]) consisting in computing a minimum spanning tree among those having $k-1$ edges, is a polynomial problem when the edge weights are bi-valued (called Min $k-M S T(a, b)$ ) whereas its maximization version $\operatorname{Max} k-\operatorname{MST}(a, b)$, also called Remote $k-M S T(a, b)$ in Halldorsson et al. [26] has been proved $N P$ - hard.

There are some tight links for these problems between differential and standard ratios. For example, most of these problems are strongly $N P$-hard and cannot have a fully polynomial time differential or standard approximation scheme unless $P=N P$. Moreover, when we deal with the case $d_{\text {max }} \leq t d_{\text {min }}$ (a case that seems to be very common in practical applications) we can establish a bridge between differential and standard ratios:

Theorem 2.4. If $\pi \in F G N P O$, then for any $t \in] 1 ; \infty],($ goal $\pi[t], \rho) \leq^{A * P}$ (goal $\left.\pi[t], \delta\right)$ with the expansion verifying:

$$
c(\varepsilon)=\frac{(t-1) \varepsilon+1}{t} \text { if goal }=\text { Max } \quad c(\varepsilon)=\frac{1}{t-(t-1) \varepsilon} \text { if goal }=\text { Min }
$$

Proof : We only demonstrate the $\operatorname{Max} \pi[t]$ case. Let $I$ be an instance and $x$ be a feasible solution. If $m[I, x] \geq \varepsilon O P T(I)+(1-\varepsilon) W O R(I)$ then $m[I, x] \geq c(\varepsilon) O P T(I)$ since $W O R(I) \geq \frac{1}{t} O P T(I)$

Observe that if $t$ tends towards infinity, we find again the lemma 1.6 for goal $=$ Max and problems of $F G N P O$, whereas for $g o a l=M i n$, the expansion is $c(\varepsilon)=0$ (in other words, we can not transfer a positive differential result to a standard result by this way). When $\pi[t]$ is $A P X-$ complete for some $t \in] 1 ; \infty[$, we can deduce from the previous theorem that the hardness thresholds for standard and differential framework are identical.

Corollary 2.5. If $\pi[t] \in F G N P O$ and is $A P X$ - complete for some $t \in] 1 ; \infty[$, then $\pi \notin P T A S(\delta)$ unless $P=N P$.

\section{The depth-constrained Minimum Spanning Tree}

In this section we consider the $f$-depth constrained spanning tree problem also called the Hop tree problem in Gouveia [23], Gouveia and Janssen [24] or the Shallow-Light spanning tree problem in Naor and Schieber[43], Kortsarz and Peleg [34], formally defined as follows. Let $f$ be an integer function, we suppose w.l.o.g. that $f(n)$ is always an integer between 2 and $n$ where $n=|V|$. 
Definition 3.1. Consider a complete graph $K_{n}$ and $r \in V$ with non-negative cost $d(e)$ for each edge $e \in E$. The problem of finding an optimal-cost spanning tree $T$ with root $r$ such that the $\operatorname{depth}^{9}$ of $T$

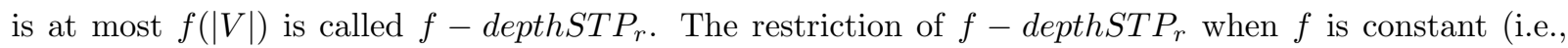
$f(n)=k \forall n)$, is called $k-\operatorname{depthSTP}$.

The Min $k$-depthSTP $P_{r}$ problem has been extensively studied by Gouveia [23], [22], [24] and arises in many applications such as telecommunication network design and facility location. It is NP-hard for any $f(n)=k \geq 2$, even if the distances are one and two (see Alfandari and Paschos [3], Manyem and Stallmann [37]) and it is generally not in $A P X$. For $k=2$, this problem is $\mathrm{O}(1 / \ln n)$-approximable under the standard ratio and is equivalent to a version of the single-source uncapacitated facility location problem whereas its restriction with distances one and two is 4/5-standard approximable and $A P X$-complete (see Alfandari and Paschos [3]). Moreover, it has been proved by Kortsarz and Peleg [34] that Min $k-\operatorname{depthSTP} P_{r}$ is $O(1 / \ln n)$-standard approximable for every constant $k \geq 2$ and Min $f-\operatorname{depthSTP} P_{r}$ is $O\left(1 / n^{\varepsilon}\right)$-standard approximable for any fixed $0<\varepsilon<1$.

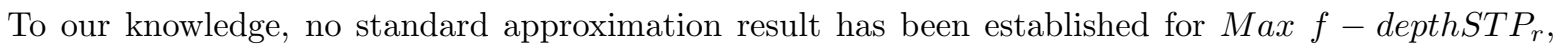
even when the depth does not depend on the graph order. Nevertheless, when the graph is directed, the bound (liminf $f-1) / \lim \inf f$ has been proved in Monnot [40]. So, the following result is presented for the sake of illustration.

We show that $\operatorname{Max} f$-depthSTP $P_{r}$ is (liminf $\left.f-1\right) / \lim \inf f$-approximable in the differential framework. Then, we deduce the same results for standard ratio.

3.1. The algorithm. For a spanning tree $T$ with root $r$ of $G=(V, E)$, let $p$ be the "father" function (i.e., $p(v)$ is the vertex adjacent to $v$ in the unique path connecting $v$ to $r$ in $T)$ of the tree and let $\left(V_{0}, V_{1}, \ldots, V_{q}\right)$ be the partition of $V$ where $V_{i}=\left\{v: r=p^{i}(v)\right\}$ is the $i$-th level vertices subset and $q$ is the depth of $T$. So, we have in particular $V_{0}=\{r\}$ and all vertices of $V_{q}$ are leaves of $T$. In order to keep it simple, we suppose that $q$ is a multiple of $f(n)$ (we could always add some $V_{i}=\emptyset$ ). Finally, let $T_{0}$ be the tree defined by $T_{0}=\cup_{v \in V \backslash\{r\}}\{(r, v)\}$, which has a depth equal to 1 . The main idea of the following algorithm consists in discarding some edges of $T$ and connecting each resulting tree to the root $r$ in order to obtain a tree with depth $f(n)$.

$\left[\right.$ shorteningtree $\left._{f}\right]$

input : A complete graph $K_{n}=(V, E)$ with $r \in V$, edge-valued by $d$

output : A tree $\operatorname{sol}_{1}$ of depth at most $f(n)$ and root $r$;

Compute a maximum weight spanning tree $T^{*}$;

Build $\left(V_{0}, V_{1}, \ldots, V_{q}\right)$ the partition associated with $T^{*}$;

For $i=1$ to $f(n)$ do

$$
\begin{aligned}
& V^{i}=\cup_{j \leq q / f(n)-1} V_{j f(n)+i} ; \\
& \text { build } T_{i}=T^{*} \cup\left\{(v, r): v \in V^{i}\right\} \backslash\left\{(v, p(v)): v \in V^{i}\right\} ;
\end{aligned}
$$

End for i

$$
\operatorname{sol}_{1}=\operatorname{argmax}\left\{d\left(T_{i}\right), i=1, \ldots, f(n)\right\} \text {; }
$$

It is easy to see that for any $i \leq f(n)$, the tree $T_{i}$ is a feasible solution and that the time-complexity of this algorithm is polynomial with respect to the encoding of the input since $f(n) \leq n$.

Theorem 3.2. The algorithm [shorteningtree $f$ ] is a $\frac{\lim \inf f-1}{\lim \inf f}$-differential approximation for Max $f-$ depthSTP $P_{r}$ and this ratio is tight.

\footnotetext{
${ }^{9}$ The depth of a tree $T$ with root $r$ is the maximum number of edges of a path in $T$ with endpoint $r$.
} 
Proof: Let $I=\left(K_{n}, d\right)$ be an instance of $\operatorname{Max} f-\operatorname{depthSTP} P_{r}$. We just have to consider the following equality:

$$
\sum_{i \leq f(n)} d\left(T_{i}\right)=(f(n)-1) d\left(T^{*}\right)+d\left(T_{0}\right)
$$

and observe that solution $\operatorname{sol}_{1}$ satisfies

$$
f(n) d\left(\operatorname{sol}_{1}\right) \geq \sum_{i \leq f(n)} d\left(T_{i}\right)
$$

On the other hand, regarding an optimal solution, we have:

$$
O P T(I) \leq d\left(T^{*}\right)
$$

and for a worst solution

$$
W O R(I) \leq d\left(T_{0}\right)
$$

Thus, using (3.1), (3.2), (3.3) and (3.4), we obtain the expected result since we are interested here only in asymptotic bounds.

Let us show that this ratio is tight: consider the following instance $I_{n}=\left(K_{n}, r, d\right)$ such that the sub-graph induced by $V \backslash\{r\}$ is a complete graph of $n \lim \inf f$ vertices and the edges-weights are two. Moreover, $d(r, v)=1, \forall v \in V \backslash\{r\}$. So, $T^{*}$ is a Hamiltonian path from $r$ to an arbitrary vertex $v$ and:

$$
d\left(\operatorname{sol}_{1}\right)=d\left(T_{1}\right)=2 n \liminf f-n, W O R\left(I_{n}\right)=n \liminf f, O P T\left(I_{n}\right)=2 n \liminf f-1
$$

Hence, we obtain $\delta_{\text {shorteningtree }_{f}}\left(I_{n}\right) \longrightarrow \frac{\liminf f-1}{\liminf f}$.

For the standard ratio, we deduce new improved results from theorem 3.2 and theorem 2.4:

Corollary 3.3. We have the following results:

- Max $f-\operatorname{depthST} P_{r}[t]$ is $\frac{t(\liminf f-1)+1}{t \lim \inf f}$-standard approximable.

- Min $f-\operatorname{depthSTP}[t]$ is $\frac{\liminf f}{\liminf f+(t-1)}$-standard approximable.

Notice that, if liminf $f$ is infinite, then $f-\operatorname{depthST} P_{r} \in P T A S(\delta), M a x f-\operatorname{depthST} P_{r} \in P T A S$ and Min $f-\operatorname{depthST} P_{r}[t] \in P T A S$ for any $\left.t \in\right] 1 ; \infty[$. In the paper Monnot [40], we improved the differential ratio to $3 / 4$ for the sub-problem $2-\operatorname{depthST} P_{r}(1,2)$ (the edge-weights are one or two and $f(n)=2, \forall n$ ) and we have derived a 4/5-standard approximation for $\operatorname{Min} 2-\operatorname{depthST} P_{r}(1,2)$ and a $7 / 8$-standard approximation for Max $2-\operatorname{depthSTP} P_{r}(1,2)$.

\section{The Strict Rural Postman Problem}

In this section we study routing problems with additional constraints on edges. Usually the traveling salesman problem is defined as finding a tour (i.e., Hamiltonian cycle) of minimal length, that a salesman can take to visit $n$ cities, each exactly once and returning to his starting city whereas the rural postman problem requires that some specified edges are traversed at least once in a solution. Hence, in the latter case the feasible solutions are not necessarily Hamiltonian cycles; it depends on the structure of the specified edges. In our version any solution must be a tour and then, we can assume that the predefined edges belong to vertex disjoint paths; indeed without loss of generality, we can suppose that the paths are shrunk to a matching. This problem called Strict Rural Postman problem and shortly noted SRPP, is a sub-case of the one described by Orloff [44] and models the problem of mail-carrier where each edge corresponds to a street along which the mail must be delivered. More generally, the rural postman problem underlies applications in contexts where roads have to be traversed for maintenance, garbage collection, school bus transportation, electrical lines and gas mains inspection, etc. like indicated in Eiselt et al. [16]. From a standard measure point of view, the metric Min SRPP is 2/3-standard approximable and the 
approximation algorithm is obtainable by using an algorithm similar to the Christofides's one [19]. To our knowledge, no standard approximation result exists for Max SRPP.

When using the differential measure, the expected results will be less optimistic, this problem containing several other particular problems which are difficult to approximate. Among those, we mention the Hamiltonian path with specified endpoints problem (denoted by $H P P_{s, p}$ ), the traveling salesman problem (in short TSP) and the clustered traveling salesman problem $^{10}$ (called $C T S P_{s_{i}, p_{i}}$ ); hence from this point of view, the problem seems to be a central routing problem. We show that this problem can be approximated with a differential ratio bounded above by $1 / 2$ and like previously, we improve the standard bounds for the case where all the edge weights are within an interval $[a, t a]$. Hence, we will deduce that $\operatorname{Min} C T S P_{s_{i}, p_{i}}[t]$, Min $H P P_{s, p}[t]$ are 2/(t+1)-standard approximable, and $\operatorname{Max} C T S P_{s_{i}, p_{i}}[t], \operatorname{Max} H P P_{s, p}[t]$ are $(t+1) / 2 t$ standard approximable.

The problem is formally defined as follows:

Definition 4.1. Consider a complete graph $K_{n}=(V, E)$ with non-negative cost $d(e)$ for each edge $e \in E$ and a matching $E^{\prime} \subset E$. The problem is to compute an optimal-cost Hamiltonian cycle of $K_{n}$ containing $E^{\prime}$. This problem is called Strict Rural postman and noted SRPP.

We exploit the structural properties of solutions to approximate this problem. The principle of our algorithm is to generate two feasible solutions following the method consisting in finding a maximum weight 2-matching among those containing $E^{\prime}$, then discarding some edges (not belonging to $E^{\prime}$ ) and arbitrarily connecting the resulting paths to form a Hamiltonian cycle. In order to build an optimal 2matching $M$ containing $E^{\prime}$, we substitute $|V| d_{\max }+1$ for the cost of each edge of $E^{\prime}$ and we compute a maximum 2-matching $M$ in this new graph. We suppose that $M$ contains cycles $\Gamma_{i}, i=1, \ldots, p$ and each cycle $\Gamma_{i}$ has at least an edge $e_{i} \in E^{\prime}$ (otherwise we take arbitrarily an edge and consider like belonging to $E^{\prime}$ ). Formally, for each cycle $\Gamma_{i}$, we always consider four consecutive vertices $x_{1}^{i}, x_{2}^{i}, x_{3}^{i}, x_{4}^{i}$, such that $\left(x_{2}^{i}, x_{3}^{i}\right) \in E^{\prime}$. Indeed, if $\left|\Gamma_{i}\right|=3$ then $x_{4}^{i}=x_{1}^{i}$ and we must have in any case $\left(x_{1}^{i}, x_{2}^{i}\right) \notin E^{\prime}$ and $\left(x_{3}^{i}, x_{4}^{i}\right) \notin E^{\prime}$.

[Patching.SRP]

input: A complete graph $K_{n}$ edge valued by $d$ and a matching $E^{\prime}$;

output : A Hamiltonian cycle $S O L$ of $G$ containing $E^{\prime}$;

Change the cost of $e \in E^{\prime}$ by $|V| d_{\max }+1$. Call $d^{\prime}$ this new weight function;

Compute a maximum weight 2-matching $M=\left\{\Gamma_{i}, i=1, \ldots, p\right\}$ of $\left(G, d^{\prime}\right)$;

if $p=1$ then $S O L=M$ and exit;

build $S_{1}=\cup_{j=1}^{p}\left\{\left(x_{1}^{j}, x_{2}^{j}\right)\right.$;

build $S_{2}=\cup_{j=1}^{p}\left\{\left(x_{3}^{j}, x_{4}^{j}\right)\right.$;

if $p$ is even then

$$
\begin{aligned}
& \text { sol }_{1}=\left[M \backslash S_{1}\right] \cup\left\{\left(x_{2}^{p-1}, x_{2}^{p}\right),\left(x_{1}^{p}, x_{1}^{1}\right)\right\} \cup_{j=1}^{(p-2) / 2}\left\{\left(x_{2}^{2 j-1}, x_{2}^{2 j}\right),\left(x_{1}^{2 j}, x_{1}^{2 j+1}\right)\right\} ; \\
& \text { sol }_{2}=\left[M \backslash S_{2}\right] \cup\left\{\left(x_{4}^{1}, x_{3}^{p}\right),\left(x_{4}^{2}, x_{3}^{p-1}\right)\right\} \cup_{j=1}^{p-2}\left\{\left(x_{3}^{j}, x_{4}^{j+2}\right)\right\} ;
\end{aligned}
$$

End if;

if $p$ is odd then

End if;

$$
\begin{aligned}
& \text { sol }_{1}=\left[M \backslash S_{1}\right] \cup\left\{\left(x_{2}^{p}, x_{1}^{1}\right)\right\} \cup_{j=1}^{(p-1) / 2}\left\{\left(x_{2}^{2 j-1}, x_{2}^{2 j}\right),\left(x_{1}^{2 j}, x_{1}^{2 j+1}\right)\right\} ; \\
& \text { sol }_{2}=\left[M \backslash S_{2}\right] \cup\left\{\left(x_{4}^{1}, x_{3}^{p-1}\right),\left(x_{4}^{2}, x_{3}^{p}\right)\right\} \cup_{j=1}^{p-2}\left\{\left(x_{3}^{j}, x_{4}^{j+2}\right)\right\} ;
\end{aligned}
$$

$S O L=\operatorname{argmax}\left\{d\left(\operatorname{sol}_{1}\right), d\left(\operatorname{sol}_{2}\right)\right\} ;$

\footnotetext{
${ }^{10}$ This problem consists in computing a tour of $K_{n}$ in which the vertices of each cluster $V_{i}$ are visited consecutively from $s_{i}$ to $p_{i}$ where $V$ is split into $V_{1}, \ldots V_{k}$.
} 
This algorithm is polynomial and we can easily observe that the solutions $\operatorname{sol}_{1}$ and $\operatorname{sol}_{2}$ are Hamiltonian cycles containing $E^{\prime}$.

Theorem 4.2. The algorithm [Patching.SRP] is a $\frac{1}{2}$-differential approximation for Max $S R P P$ and this ratio is tight.

Proof: Let $I=\left(K_{n}, E^{\prime}, d\right)$ be an instance and let $S O L^{*}$ be an optimal Hamiltonian cycle containing $E^{\prime}$. We denote $\operatorname{loss}_{i}, i=1,2$, the quantity $d\left(\right.$ sol $\left._{i}\right)-d(M)$. Obviously, loss $_{i} \leq 0$ and we have

$$
d(S O L) \geq d(M)+\frac{1}{2}\left(\operatorname{loss}_{1}+\operatorname{loss}_{2}\right)
$$

Moreover, the following structural property holds:

$$
S O L_{*}=\cup_{j=1,2}\left(\operatorname{sol}_{j} \backslash M\right) \cup M \backslash\left(S_{1} \cup S_{2}\right) \text { is a Hamiltonian cycle containing } E^{\prime}
$$

Hence, $S O L_{*}$ is a feasible solution of our problem and approximates well the value of a worst solution. Indeed, when $\operatorname{sol}_{i}$ is better than $\operatorname{sol}_{3-i}$ then from $s o l_{3-i}$ we can build a tour (the solution $S O L_{*}$ ) which is relatively worst. The cost of this solution is $d(M)+\operatorname{loss}_{1}+\operatorname{loss}_{2}$ since we have $d\left(\operatorname{sol}_{j}\right)=l o s s_{j}+d(M)$ and $d\left(M \backslash\left(S_{1} \cup S_{2}\right)\right)=d\left(M_{r^{*}}\right)-d\left(S_{1}\right)-d\left(S_{2}\right)$. Thus, we deduce

$$
W O R(I) \leq d(M)+\operatorname{loss}_{1}+\operatorname{loss}_{2}
$$

Since $S O L^{*}$ is a particular 2-matching containing $E^{\prime}$, we have:

$$
O P T(I) \leq d(M)
$$

Finally, combining (4.1), (4.3) and (4.4) we obtain:

$$
d(S O L) \geq \frac{1}{2} W O R(I)+\frac{1}{2} O P T(I)
$$

To show that the bound is tight, consider the following instances $I_{n}=\left(K_{n}, E^{\prime}, d\right)$ with $V=\left\{x_{i}^{j}, 1 \leq j \leq\right.$ $2 n, 1 \leq i \leq 4\}, E^{\prime}=\left\{\left(x_{2}^{j}, x_{3}^{j}\right),\left(x_{4}^{j}, x_{1}^{j}\right), 1 \leq j \leq 2 n\right\}, d\left(x_{1}^{j}, x_{2}^{j}\right)=d\left(x_{2}^{j}, x_{3}^{j}\right)=d\left(x_{3}^{j}, x_{4}^{j}\right)=d\left(x_{4}^{j}, x_{1}^{j}\right)=2$, $d\left(x_{1}^{2 j-1}, x_{1}^{2 j}\right)=d\left(x_{2}^{2 j}, x_{2}^{2 j+1}\right)=2, j=1, \ldots, n-1$, and $d\left(x_{1}^{2 n-1}, x_{1}^{2 n}\right)=d\left(x_{2}^{2 n}, x_{2}^{1}\right)=2$. Let the cost of all other edges be one. Thus, we obtain:

and thus $\delta_{\text {Patching.SRP }}\left(I_{n}\right)=\frac{1}{2}$.

$$
d(S O L)=3 n, W O R\left(I_{n}\right)=2 n, O P T\left(I_{n}\right)=4 n
$$

4.1. Some links with other routing problems. Now, we study some particular cases of this problem which are known to be $N P$-hard problems in the literature. For instance, the traveling salesman problem can be viewed as a special case of $S R P P$ in which there is no specified edge (take $\left.E^{\prime}=\emptyset\right)$. Nevertheless, this problem is as well approximable as $T S P$ in its minimization metric version since there is a $2 / 3$-standard approximation and it is the best known ratio until today.

Since we have proved in Monnot et al. [41] that no polynomial time algorithm can guarantee a differential approximation ratio greater than $649 / 650$ for $T S P[6]$ and $741 / 742$ for $T S P[2]$, we can deduce the following negative results:

Proposition 4.3. If $P \neq N P$ then:

- For any $t \in\left[2 ; 6\left[, S R P P[t]\right.\right.$ is not approximable with differential ratio greater than $\frac{741}{742}$

- For any $t \in[6 ; \infty], S R P P[t]$ is not approximable with differential ratio greater than $\frac{649}{650}$

This problem also contains Hamiltonian path problem with two specified endpoints and clustered traveling salesman problem with specified endpoints; the first one consists in finding, in a complete graph $K_{n}$ valuated on edges, a Hamiltonian path from $s$ to $p$ of optimum weight whereas the second one consists in computing a tour of $K_{n}$ in which the vertices of each cluster $V_{i}$ are visited consecutively from $s_{i}$ to $p_{i}$, where $V$ is 
split into $V_{1}, \ldots V_{k}$. These properties seem not to hold for the standard framework; when considering the minimization metric version, several authors estimate that $H P P_{s, p}$ and $C T S P_{s_{i}, p_{i}}$ are more difficult to approximate than TSP or SRPP. Already in the middle of the eighties, Johnson and Papadimitriou [31] raised the question of the relative hardness of the Hamiltonian path specified endpoint version compared to the traveling salesman. These conjectures are strengthened by the positive results given on these problems since the best-known standard ratios are respectively $3 / 5$ and $11 / 21$ for Min metric $-H P P_{s, p}$ (see Hoogeveen [30]) and Min metric - CTS $P_{s_{i}, p_{i}}$ (see Guttmann-Beck et a. [25]) whereas it is $2 / 3$ for Min metric - SRPP.

Proposition 4.4. The following results hold:

(i) $\left(H P P_{s, p}, \delta\right) \leq{ }^{A * P}(S R P P, \delta)$ with $c(\varepsilon)=\varepsilon$.

(ii) $\left(C T S P_{s_{i}, p_{i}}, \delta\right) \leq{ }^{A * P}(S R P P, \delta)$ with $c(\varepsilon)=\varepsilon$.

Proof: Suppose that $S R P P$ contains a solution $s o l^{\prime}$ which is an $\varepsilon$-differential approximation.

For (i) When $E^{\prime}=\{(s, p)\}$, sol' is a tour traversing $(s, p)$. Thus, the removal of this edge forms a Hamiltonian path from $s$ to $p$ (noted by $s o l$ ) and we have $d(s o l)=d\left(s o l^{\prime}\right)-d(e)$. Moreover, the same property holds for the respective optimum and the respective worst values, so we obtain:

$$
\begin{aligned}
d(s o l) & =d\left(s o l^{\prime}\right)-d(e) \\
d(s o l) & \leq \varepsilon\left(O P T_{S R P P}(I)-d(e)\right)+(1-\varepsilon)\left(W O R_{S R P P}(I)-d(e)\right) \\
d(s o l) & \leq \varepsilon O P T_{H P P}(I)+(1-\varepsilon) W O R_{H P P}(I)
\end{aligned}
$$

For (ii): Let $I=\left(K_{n}, d\right)$ be an instance of $\operatorname{Min} C T S P_{s_{i}, p_{i}}$ such that $\left(V_{1}, \ldots, V_{k}\right)$ is a partition of $V$ and $\left\{s_{i}, p_{i}\right\} \subseteq V_{i}$. Denote by $G_{i}$ the sub-graph of $K_{n}$ induced by $V_{i}$ and $G^{\prime}$ the sub-graph of $K_{n}$ induced by $\left\{s_{i}, p_{i} \mid 1 \leq i \leq k\right\}$. Consider $I_{i}=\left(G_{i}, d, s_{i}, p_{i}\right)$ and $I^{\prime}=\left(G^{\prime}, d, E^{\prime}\right)$ with $E^{\prime}=\left\{\left(s_{i}, p_{i}\right), 1 \leq i \leq k\right\}$ as being respectively an instance of $H P P_{s, p}$ and an instance of $S R P P$.

From (i), we know that $H P P_{s, p}$ is $\varepsilon$-differential approximable and if $s o l_{i}$ (resp. $s o l^{\prime}$ ) is a feasible solution for $H P P_{s_{i}, p_{i}}$ on $I_{i}$ (resp. $S R P P$ on $I^{\prime}$ ), then $s o l=\left(\operatorname{sol}^{\prime} \backslash E^{\prime}\right) \cup_{i=1, \ldots, k} \operatorname{sol}_{i}$ is a feasible solution of $I$ for $C T S P_{s_{i}, p_{i}}$; this same partition of optimal solutions also hold and we have:

$$
\begin{aligned}
d(s o l) & =d\left(s^{\prime} l^{\prime}\right)-d\left(E^{\prime}\right)+\sum_{i=1}^{k} d\left(\operatorname{sol}_{i}\right) \\
d(s o l) & \leq \varepsilon\left(O P T_{S R P P}\left(I^{\prime}\right)-d\left(E^{\prime}\right)\right)+(1-\varepsilon)\left(W O R_{S R P P}\left(I^{\prime}\right)-d\left(E^{\prime}\right)\right) \\
& +\sum_{i=1}^{k}\left[\varepsilon O P T_{H P P}\left(I_{i}\right)+(1-\varepsilon) W O R_{H P P}\left(I_{i}\right)\right] \\
d(s o l) & \leq \varepsilon O P T_{C T S P}(I)+(1-\varepsilon) W O R_{C T S P}(I)
\end{aligned}
$$

That is the expected result.

We deduce from the previous results, thanks to theorem 2.4 and lemma 1.6:

Theorem 4.5. For any $t \in] 1 ; \infty]$, we have the following results:

- Min $C T S P_{s_{i}, p_{i}}[t]$, Min HPP $P_{s, p}[t]$ and Min SRPP $[t]$ are $\frac{2}{t+1}$-standard approximable.

- $\operatorname{Max} C T S P_{s_{i}, p_{i}}[t], \operatorname{Max} H P P_{s, p}[t]$ and Max $S R P P[t]$ are $\frac{t+1}{2 t}$-standard approximable.

\section{Conclusion}

In this article, we have established some basic properties on the approximability of FGNPO-problems with respect to the differential ratio. We also have exposed positive approximation results for some specific vehicles routing and spanning tree problems.

An open question of particular interest regarding to this class is the following: does any FGNPOproblem accept a differential approximation scheme? We conjecture that the answer is no, due to the structural properties on the size of any feasible solution that define FGNPO problems.

Moreover, for a class of problems close to FGNPO, namely when replacing the value $m\left[I, E^{\prime}\right]=$ $\sum_{e \in E^{\prime}} d(e)$ of any solution $E^{\prime}$ by $m\left[I, E^{\prime}\right]=\max _{e \in E^{\prime}} d(e)$ and possibly turn the goal to minimize, the 
negative results become stronger. Actually, we denote by $N O A P X(\Delta)$ the class of problems $\pi$ from $N P O$ which are not differential approximable better than 0 by any polynomial time algorithm, unless $P=N P$ (even when looking for a ratio depending on the size of the instance). In other words, NOAPX( $\Delta)$ is constituted by the problems $\pi$ which admit, for any polynomial time approximate algorithm $A$, an instance $I_{0}$ such that $\delta_{A}\left(I_{0}\right)=0$. Thus, this class contains the worst approximation results we may expect (with respect to this measure), but still we have shown in Monnot et al [42] that both the $\{0,1\}$-Linear Programming problem (with goal to minimize as well as to maximize) and a special weighted satisfiability problem are one of them. Now regarding to $F G N P O$, we have the following result, given in Monnot [39], similar to the proposition 2.2 transposed to the differential framework.

Let $\pi$ be an FGNPO problem and $\pi^{\prime}$ derived from $\pi$ with the evaluating function given by $m\left[I, E^{\prime}\right]=$ $\max _{e \in E^{\prime}} d(e)$ for any solution $E^{\prime}$ for $\pi$. If the decision problem associated to Min $\pi^{\prime}$ is $N P$-Complete, then $\operatorname{Min} \pi \in N O A P X(\Delta)$.

\section{REFERENCES}

[1] A. Aggarwal, H. Imai, N. Katoh, and S. Suri. Finding $k$ points with minimum diameter and related problems. $J$. Algo., 12:38-56, 1991

[2] A. Aiello, E. Burattini, M. Massarotti, and F. Ventriglia. A new evaluation function for approximation. Sem. IRIA., 1977.

[3] L. Alfandari and V. Th. Paschos. Approximating the minimum rooted spanning tree with depth two. Int. Transactions in Oper. Res., 6:607-622, 1999.

[4] G. Ausiello, P. Crescenzi, G. Gambosi, V. Kann, A. Marchetti Spaccamela, and M. Protasi. Complexity and approximation: Combinatorial optimization problems and their approximability properties. Springer Verlag, 1999.

[5] G. Ausiello, P. Crescenzi, and M. Protasi. Approximate solutions of NP-optimization problems. Theo. Comp. Sci., 150:1-55, 1995.

[6] G. Ausiello, A. D'Atri, and M. Protasi. Structure preserving reductions among convex optimization problems. $J$. Comp. Sys. Sci., 21:136-153, 1980.

[7] N. Christofides. Worst-case analysis of a new heuristic for the traveling salesman problem. Technical report 338 Grad School of Indistrial Administration, CMU, 1976

[8] G. Cornuejols, M. L. Fisher, and G. L. Memhauser. Location of bank accounts to optimize float: An analytic study of exact and approximate algorithms. Manag. Sci., 23:789-810, 1977.

[9] P. Crescenzi and V. Kann. A compendium of NP-optimization problems. Available on www address: http://www.nada.kth.se/ viggo/problemlist/compendium.html, 1998.

[10] P. Crescenzi and A. Panconesi. Completness in approximation classes. Inf. and Comp., 93:241-262, 1991.

[11] M. Demange, D. de Werra, J. Monnot, and V. Th. Paschos. Weighted node coloring: when stable sets are expensive (extended abstract). Proc. W.G. (to appear), 2002.

[12] M. Demange, P. Grisoni, and V. Th. Paschos. Differential approximation algorithms for some combinatorial optimization problems. Theo. Comp. Sci., 209:107-122, 1998.

[13] M. Demange, J. Monnot, and V. Th. Paschos. Bridging gap between standard and differential polynomial approximation: the case of bin-packing. Appl. math. Let., 12:127-133, 1999.

[14] M. Demange, J. Monnot, and V. Th. Paschos. Differential approximation results for the Steiner tree problem. Appl. math. Let. (to appear), 2002

[15] M. Demange and V. Th. Paschos. On an approximation measure founded on the links between optimization and polynomial approximation theory. Theo. Comp. Sci., 156:117-141, 1996.

[16] H. A. Eiselt, M. Gendreau, and G. Laporte. Arc routing problems, part II: the rural postman problem. Op. Res. (Survey, Expository and Tutorial), 43:399-414, 1995.

[17] L. Engebretsen and M. KARPInski. Approximation hardness of TSP with bounded metrics. ECCC Report TR00-089,, 2000.

[18] M. L. Fisher, G. L. Nemhauser, and L. A. Wolsey. An analysis of approximations for finding a maximum weight hamiltonian circuit. Op. Res., 27(4):799-809, 1979.

[19] G. N. Frederickson. Approximation algorithm for some postman problems. J. ACM, 26:538-554, 1979.

[20] M. R. Garey and D. S. Johnson. Computers and intractability. A guide to the theory of NP-completeness. CA. Freeman, 1979.

[21] N. Garg. A 3-approximation for the minimum tree spanning $k$ vertices. In Proc. FOCS, pages 302-309, 1996. 
[22] L. Gouveia. Multicommodity flow models for spanning tree with hop constraints. European J. Oper. Res., 95:178-190, 1996.

[23] L. Gouveia. Using variable redefinition for computing lower bounds for minimum spanning and Steiner trees with hop constraints. J. Comp., 10(2):180-188, 1998.

[24] L. Gouveia and E. Janssen. Designing reliable tree with two cable technologies. European J. Oper. Res., 105:552-568, 1998.

[25] N. Guttmann-Beck, R. Hassin, S. Khuller, and B. Raghavachari. Approximation algorithms with bounded performance guarantees for the clustered traveling salesman problem. Algorithmica, 4:422-437, 2000.

[26] M. M. Halldorsson, K. Iwano, N. Katoh, and T. Tokuyama. Finding subsets maximizing minimum structures. In Proc. SODA, pages 150-159, 1995.

[27] R. Hassin and S. Khuller. z-approximations. J. Algo., 41:429-442, 2002.

[28] R. Hassin and S. Rubinstein. An approximation algorithm for maximum packing of 3-edge paths. Inf. Proc. Let, 6:63-67, 1997.

[29] D. Hochbaum, Editor. Approximation algorithms for NP-hard problems. P.W.S, 1997.

[30] J. A. Hoogeveen. Analysis of christofides' heuristic: Some paths are more difficult than cycles. Oper. Res. Let., 10:291295, 1991.

[31] D. S. Johnson and C. H. Papadimitriou. Performance guarantees for heuristics. E. L LAwler, J. K. Lenstra, A. H. G. Rinnooy Kan And D. B. Shmoys (EDs) The Traveling Salesman Problem: a guided tour of Combinatorial Optimization WiLey Chichester 145 - 180, 1985.

[32] R. M. KARP. Reducibility among combinatorial problems. R.E MiLleR and J.W. ThatcheR editors, Complexity of Computer Computations, 85-103, Plenum Press, NY, 1972.

[33] D. G. Kirkpatrick and P. Hell. The complexity of a generalized matching problem. In Proc. STOC, pages 240-245, 1978.

[34] G. Kortsarz and D. Peleg. Approximating shallow-light trees. In Proc. SODA, pages 103-110, 1997.

[35] S. R. Kosaraju, J. K. Park, and C. Stein. Long tours and short superstrings. In Proc. FOCS, pages 166-177, 1994.

[36] T. Magnanti and L. Wolsey. Optimal trees. Network models, Handbooks in Oper. Res. and Manag. sci., 7:503-615, 1995.

[37] P. Manyem and M. F. M Stallmann. Some approximation results in multicasting. Working paper, North Carolina State University, 1996.

[38] J. S. B. Mitchell. Guillotine subdivisions approximate polygonal subdivisions: Part II-a simple polynomial-time approximation scheme for geometric TSP, k-MST, and related problems. SIAM J. Comp., 28:1298-1309, 1999.

[39] J. Monnot. Families of critical instances and polynomial approximation. PhD thesis, LAMSADE, Université Paris IX, Dauphine, 1998 (in French).

[40] J. Monnot. The maximum f-depth spanning tree problem. Inf. Proc. Let, 80(4):179-187, 2001.

[41] J. Monnot, V. Th. Paschos, and S. Toulouse. Differential approximation results for traveling salesman problem with distance 1 and 2 (extended abstract). Proc. FCT, 2138:275-286, 2001.

[42] J. Monnot, V. Th. Paschos, and S. Toulouse. Approximation polynomiale des problèmes NP-difficiles: optima locaux et rapport différentiel. Edition HERMES Science Lavoisier, 2003.

[43] J. S. NAOR and B. SCHIEBER. Improved approximations for shallow-light spanning trees. In Proc. FOCS, pages 536-541, 1997.

[44] C. S. Orloff. A fundamental problem in vehicle routing. Networks, 4:35-64, 1974.

[45] P. Orponen and H. Mannila. On approximation preserving reductions: complete problems and robust measures. Technical report C-1987-28, Department of Computer Science, University of Helsinki, 1987.

[46] R. Ravi, R. Sundaram, M. V. Marathe, D. J. Rosenkrants, and S. S. Ravi. Spanning tree short or small. SIAM J. Disc. Math., 9:178-200, 1996.

[47] S. SAhni and T. Gonzalez. P-complete approximation problems. J. ACM, 23:555-565, 1976.

[48] S. A. VAvasis. Approximation algorithms for indefinite quadratic programming. Math. Prog., 57:279-311, 1972.

[49] E. ZEMEL. Measuring the quality of approximate solutions to zero-one programming problems. Math. Op. Res., 6:319332,1981 . 